\title{
Ventilatory requirements of quadriceps resistance training in people with COPD and healthy controls
}

This article was published in the following Dove Press journal:

International Journal of COPD

5 June 2014

Number of times this article has been viewed

\author{
Linzy Houchen-Wolloff' \\ Carolyn J Sandland' \\ Samantha L Harrison \\ Manoj K Menon' \\ Mike D Morgan' \\ Michael C Steiner \\ Sally J Singh ${ }^{1,2}$ \\ 'Centre for Exercise and \\ Rehabilitation Science, Respiratory \\ Biomedical Research Unit, Glenfield \\ Hospital, Leicester, UK; ${ }^{2}$ Faculty of \\ Health and Life Sciences, Coventry \\ University, Coventry, UK
}

Background: It is proposed that resistance training (RT) does not activate the cardiopulmonary system to the same extent as whole-body exercise. This is important for patients with chronic obstructive pulmonary disease (COPD) who are ventilatory limited.

Objective: The aim was to assess the ventilatory response to an isokinetic quadriceps RT program in people with COPD and healthy controls.

Design: Observational.

Registration number: ISRCTN22764439.

Setting: Outpatient, university teaching hospital.

Participants and outcome measures: People with COPD ( $\mathrm{n}=14)$ and healthy controls $(n=11)$ underwent breath-by-breath analysis of their ventilation during an RT session (five sets of 30 maximal knee extensions at $180^{\circ} / \mathrm{sec}$ ). Subjects performed a maximal cycle ergometry test (CET) at baseline. Peak ventilation (VE; L/min) and oxygen consumption $\left(\mathrm{VO}_{2} ; \mathrm{mL} / \mathrm{kg} / \mathrm{min}\right)$ were collected. The same system measured $\mathrm{VO}_{2}$ and VE during the RT session. Parameters are presented as a percentage of the maximal CET. Isokinetic workload, symptom scores, heart rate (HR), and oxygen saturation were documented post-training.

Results: People with COPD worked at higher percentages of their maximal capacity than controls (mean range between sets $1-5$ for $\mathrm{VO}_{2}=49.1 \%-60.1 \%$ [COPD], $45.7 \%-51.43 \%$ [controls] and for $\mathrm{VE}=57.6 \%-72.2 \%$ [COPD], 49.8\%-63.6\% [controls]), although this was not statistically significant $(P>0.1$ in all cases). In absolute terms, the difference between groups was only significant for actual $\mathrm{VO}_{2}$ on set $2(P<0.05)$. Controls performed more isokinetic work than patients with COPD $(P<0.05)$. Median Borg symptom scores after RT were the same in both groups ( 3 breathlessness, 13 exertion), no de-saturation occurred, and both groups were training at $\geq 65 \%$ of their maximum $\mathrm{HR}$.

Conclusion: No statistically significant differences were found between people with COPD and healthy controls for $\mathrm{VO}_{2}$ and $\mathrm{VE}$ achieved during training. The symptoms associated with training were within acceptable limits.

Keywords: exercise, ventilation, strength training, resistance training

\section{Introduction}

Skeletal muscle dysfunction (muscle weakness and wastage) is a key feature of chronic obstructive pulmonary disease (COPD) and may be related to deconditioning, chronic inflammation, and medication use. Resistance training (RT) can improve muscle mass and strength in people with COPD. ${ }^{1,2}$ Increases in quadriceps strength are well documented, ${ }^{3}$ and improvements after training are generally in the region of $20 \% .^{2,4}$ RT may offer advantages in people with severe disease over whole-body exercise, where ventilatory limitation is often the main contributor to exercise intolerance,
Correspondence: Linzy Houchen-Wolloff Centre for Exercise and Rehabilitation Science, Respiratory Biomedical Research Unit, Glenfield Hospital, Groby Road, Leicester, LE3 9QP, UK

Tel +44 II6 2583035

Fax +44 II 62502743

Email linzy.houchen@uhl-tr.nhs.uk or linzyhouchen@hotmail.com 
ie, participants terminate exercise because they are unable to increase ventilation in response to increasing metabolic demands. ${ }^{5}$

There is a school of thought that, for people with COPD, RT of smaller muscle volumes (ie, the quadriceps vs the whole body) may allow for higher training intensities at a muscle level than traditional whole-body exercise. ${ }^{6}$ Probst et $\mathrm{al}^{7}$ have shown that cardiopulmonary stress and metabolic load is lower in resistance exercise than in endurance training at the start, middle, and end of a 12-week rehabilitation program. This was observed in both objective (eg, minute ventilation, oxygen uptake derived from a portable breathby-breath system) and subjective (ie, symptom scores) parameters. Subjects also experienced less dyspnea during leg press exercise than during cycling (weeks 1, 6, and 12; $P<0.05)$.

More recently, Sillen et $\mathrm{al}^{8}$ found that the metabolic response was significantly lower still with neuromuscular electrical stimulation (NMES) than with RT during a single session. The authors concluded that both NMES and RT resulted in acceptable metabolic responses and symptom scores for participants. For instance, peak oxygen uptake (as a percentage of the maximum from cardiopulmonary exercise testing) was $57 \%$ for RT and 34\% for NMES $(P<0.001$ between RT and NMES), and Borg dyspnea scores were 3 (RT) and 1 (NMES) $(P<0.01$ between groups).

Isokinetic dynamometry represents the gold standard in the observation of muscle performance for testing and training. ${ }^{9}$ The unique RT program used in this study was based upon some novel research conducted at the University of Nottingham in 2004. ${ }^{10}$ The authors found that by immobilizing healthy young men in a limb cast for 2 weeks, a 5\% decrease in quadriceps mass occurred. Following the carefully controlled isokinetic RT program at $180^{\circ} / \mathrm{sec}$, muscle mass and strength returned to basal levels after 6 weeks. The rapid training velocity that was chosen targets type II muscle fibers and was found to be feasible and acceptable to our frail patients in a pilot study. ${ }^{11}$

Whilst the two aforementioned studies measured the cardiorespiratory response to conventional quadriceps RT (multigym/isotonic training), the response has not been explored for isokinetic training. Isokinetic testing is rarely used in the evaluation of strength for patients with COPD ${ }^{12}$ and has not been used as an RT device in this population. Neither of the previous studies ${ }^{7,8}$ have compared the ventilatory response in patients with COPD with those of healthy controls trained in an identical fashion. The current study is novel, as the results in people with COPD have reference to a healthy control group. Furthermore the ventilatory response during isokinetic training has not previously been presented.

\section{Purpose}

The purpose of this study was to determine the breath-bybreath response to one isokinetic RT session by measuring parameters of ventilation and gas exchange (vs a maximal cycle ergometry test [CET]) in participants with COPD and healthy controls.

\section{Methods}

\section{Patients}

People with COPD were recruited from outpatient clinics at Glenfield Hospital (Leicester, UK) and from those on the waiting list for pulmonary rehabilitation. COPD was documented by a physician and confirmed by spirometry. Healthy controls of a similar age were recruited via local advertisement. All participants were taking part in a larger randomized controlled trial (RCT) investigating the effects of 8 weeks lower-limb RT in people with COPD and comparing the response with that of age-matched controls. ${ }^{13}$ None of the subjects had been previously taking part in any regular exercise programs, and COPD patients who underwent pulmonary rehabilitation in the last 12 months were excluded. Other exclusion criteria included maintenance oral corticosteroid therapy, long-term oxygen therapy, diabetes, or other comorbid conditions that would prevent exercise training. The study was approved by the Leicestershire and Rutland Research Ethics Committee (REC reference 06/Q2501/138), and all participants provided written informed consent.

A convenience sample from the larger trial ${ }^{13}$ volunteered to undergo additional breath-by-breath analysis of their ventilation and gas exchange during one of their training sessions (five sets of 30 maximal knee extensions). This included 14 participants with COPD and 11 healthy controls. At baseline, spirometry was measured in all subjects in a seated position (Model R; Vitalograph ${ }^{\circledR}$, Buckingham, UK) according to accepted standards. ${ }^{14}$

\section{Whole-body exercise performance}

All subjects performed a symptom-limited, maximal, incremental CET prior to starting the RT program. A mouthpiece with flow sensor, collected breath-by-breath measurements of gas exchange and ventilation during the CET. Specifically, resting and peak measures of VE $(\mathrm{L} / \mathrm{min}), \mathrm{VO}_{2}(\mathrm{~mL} / \mathrm{kg} / \mathrm{min})$, and work (W). The ergospirometry system used was the ZAN 600 ErgoTest (ZAN Meßgeräte GmbH, Oberthulba, Germany). 
Resting and peak measures of heart rate (HR), blood pressure (BP), oxygen saturation $\left(\mathrm{SpO}_{2}\right)$, Borg breathlessness levels, and Borg perceived exertion (RPE) levels ${ }^{15}$ were also collected. A ramp protocol was utilized, ensuring a steady linear increase in load over time. The increase was $10 \mathrm{~W} / \mathrm{min}$ for participants with COPD and $20 \mathrm{~W} / \mathrm{min}$ for healthy subjects, after an initial warm-up period (no load). Subjects were asked to cycle at a cadence of 40-50 revolutions per minute (RPM), which was visible to them. The test ended when the subject could no longer continue due to symptom provocation or if they were unable to maintain the required speed.

\section{RT session}

Training prescription was the same for both groups and took place using an isokinetic dynamometer (Cybex II Norm; Computer Sports Medicine Inc., Stoughton, MA, USA). The knee/hip adaptor pad was strapped to the distal part of the tibia at approximately $5 \mathrm{~cm}$ above the lateral malleolus of the ankle. The range of movement was set between $10^{\circ}$ and $80^{\circ}$ flexion. Subjects performed five sets of 30 maximal knee extensions; the contractions were isokinetic and concentric at a speed of $180^{\circ} / \mathrm{sec}$. Maximal knee extension effort was chosen in an attempt to ensure a high proportion of muscle fiber recruitment, ${ }^{10}$ and volunteers were verbally encouraged at all times to elicit maximal effort. Each set was separated by a 1 minute rest, and both legs were trained (approximately 10 min training time per leg). Training workload (cumulative over five training sets) was recorded in joules (J).

During a single RT session, subjects wore a mouthpiece attached to the same ergospirometry system used for the CET. This investigation took place around the midpoint of the training program (between sessions 8-12 of 24 sessions) for both groups, and the five training sets on the dominant (right) leg were chosen for analysis. The recorded outcomes were as follows: peak oxygen uptake $\left(\mathrm{VO}_{2}: \mathrm{mL} / \mathrm{kg} / \mathrm{min}\right)$ and minute ventilation (VE: L/min) for each individual, on each of the five RT sets. These values were compared with the baseline CET values. Before and after the five sets, Borg breathlessness and RPE levels were recorded, along with $\mathrm{HR}$ and $\mathrm{SpO}_{2}$.

\section{Statistical analysis}

The sample size in the larger trial was estimated from our pulmonary rehabilitation program. We anticipated that a $20 \%$ increase in strength following training would be clinically and physiologically significant. To detect this strength difference ( $80 \%$ power, $\alpha=0.05$ ), we required 25 patients to complete training in each group. ${ }^{13}$ However, the data presented in this paper represent a convenience sample of 14 people with COPD and 11 healthy controls.

Statistical analysis was performed using the statistical package for the Social Sciences (SPSS) version 16.0 (and later version 17.0, PASW) (SPSS Inc., Chicago, IL, USA). The level of statistical significance was set at $P<0.05$. The data were checked for normality prior to statistical test selection.

The ventilation outcomes ( $\mathrm{VO}_{2}$ and $\mathrm{VE}$ achieved) during the single RT session are presented as a percentage of the maximal CET at baseline. Actual values achieved during each set are also reported. The workload $(\mathrm{J})$ performed during training and symptom scores were also analyzed in both groups. Independent and paired $t$-tests were used to look at differences between and within groups, respectively. To examine differences between groups for Borg scores (ordinal), a Mann-Whitney $U$ test was employed. A repeated measures analysis of variance (ANOVA) was used to look at the differences between sets 1 and 5 for $\mathrm{VO}_{2}$ and $\mathrm{VE}$, within-groups.

\section{Results}

Table 1 outlines the baseline characteristics of the two groups. In the COPD group, $50 \%$ of subjects were male, and $30 \%$ were male in the healthy control group (these differences were not statistically significant within or between the groups, $P>0.05$ ).

During the RT session, controls achieved an average quadriceps work load over the five training sets of 1,032.6 J compared with $750.9 \mathrm{~J}$ in the people with COPD. This difference was statistically significant $(P<0.05)$.

Table 2 shows the mean ( \pm standard deviation $[\mathrm{SD}]$ ) percentage of $\mathrm{VO}_{2}(\mathrm{~mL} / \mathrm{kg} / \mathrm{min})$ and $\mathrm{VE}(\mathrm{L} / \mathrm{min})$ achieved

Table I Baseline characteristics of the two groups

\begin{tabular}{lll}
\hline Characteristic & $\begin{array}{l}\text { COPD } \\
(\mathbf{n}=14)\end{array}$ & $\begin{array}{l}\text { Control } \\
(\mathbf{n}=\mathrm{I} \mathbf{I})\end{array}$ \\
\hline Age (years) & $71.5(7.5)$ & $65.5(5.7)^{*}$ \\
$\mathrm{BMI}$ & $26.5(4.5)$ & $27.5(2.4)$ \\
$\mathrm{FEV}_{1}(\mathrm{~L})$ & $1.0(0.5)$ & $2.5(0.9)^{* *}$ \\
$\mathrm{FEV}_{1} / \mathrm{FVC}$ ratio & $38.8(\mathrm{II} .9)$ & $73.5(5.5)^{* *}$ \\
Isometric strength $(\mathrm{Nm})$ & $104.5(42.8)$ & $132.4(46.4)$ \\
CET workload $(\mathrm{W})$ & $54.5(18.6)$ & $1 \mathrm{I} 4.1(40.6)^{* *}$ \\
CET peak $\mathrm{VO}_{2}(\mathrm{~mL} / \mathrm{kg} / \mathrm{min})$ & $15.2(4.4)$ & $21.5(5.7)$ \\
CET peak $\mathrm{VE}(\mathrm{L} / \mathrm{min})$ & $30.5(10.3)$ & $42.8(16.0)$ \\
\hline
\end{tabular}

Notes: Values are mean (SD). $* P<0.05, * * P<0.01$ between COPD and control subjects.

Abbreviations: BMI, body mass index; CET, cycle ergometry test; COPD, chronic obstructive pulmonary disease; $\mathrm{FEV}_{1}$, forced expiratory volume in I second; FVC, forced vital capacity; SD, standard deviation; VE, ventilation; $\mathrm{VO}_{2}$, oxygen consumption. 
Table 2 The percentage of maximum oxygen consumption and ventilation achieved for sets $\mathrm{I}-5$, during one isokinetic resistance training session, compared with maximal cycle ergometry testing at baseline

\begin{tabular}{cll}
\hline & COPD & Healthy control \\
\hline $\begin{array}{l}\text { \% } \\
\text { Set I }\end{array}$ achieved & $49.1(16.3)$ & $45.7(15.3)$ \\
Set 2 & $54.8(16.9)^{*}$ & $50.4(13.9)$ \\
Set 3 & $58.9(17.6)$ & $52.2(14.3)$ \\
Set 4 & $60.0(17.9)$ & $50.4(14.3)$ \\
Set 5 & $60.1(17.7)$ & $51.4(10.5)$ \\
\%VE achieved & & \\
Set I & $57.6(16.7)$ & $49.8(17.7)$ \\
Set 2 & $66.1(19.2)$ & $62.1(15.7)$ \\
Set 3 & $70.3(17.3)$ & $65.3(17.7)$ \\
Set 4 & $71.6(19.9)$ & $62.6(15.7)$ \\
Set 5 & $72.2(20.5)$ & $63.6(17.7)$ \\
\hline
\end{tabular}

Notes: All values are mean (SD). $* P<0.05$ between sets $\mathrm{I}$ and 2 for the $\% \mathrm{VO}_{2}$ achieved in patients with COPD.

Abbreviations: COPD, chronic obstructive pulmonary disease; SD, standard deviation; VE, ventilation; $\mathrm{VO}_{2}$, oxygen consumption.

in each set during a single RT session, in participants with COPD and healthy controls. These values are presented as a percentage of the maximum achieved on the baseline CET. For people with COPD, the percentage of $\mathrm{VO}_{2}$ and VE increased with each set (from mean $49.1 \%$ to $60.1 \%$ for $\mathrm{VO}_{2}$ and from mean $57.6 \%$ to $72.2 \%$ for $\mathrm{VE}$ ). $\mathrm{Th}^{\mathrm{VO}}$ increase was significant between sets 1 and 2 (mean 49.1\% to $54.8 \% ; P<0.05)$. Control subjects worked at the highest percentage of their $\mathrm{VO}_{2}$ and $\mathrm{VE}$ on set 3 (mean $52.3 \%$ for $\mathrm{VO}_{2}, 65.3 \%$ for $\left.\mathrm{VE}\right)$, despite training workload $(\mathrm{J})$ being significantly lower than in training sets 1 and $2(P<0.01)$. Overall, people with COPD worked at higher percentages of their maximal $\mathrm{VO}_{2}$ and $\mathrm{VE}$ did controls during all five sets; although this was not statistically significant $(P>0.1$ in all cases). The $\mathrm{VO}_{2}$ and $\mathrm{VE}$ achieved during RT was significantly lower than the maximum values achieved during the baseline CET (shown in Table 1; $P<0.05$ for both groups, both outcomes).

Figure 1 shows the absolute $\mathrm{VO}_{2}$ and $\mathrm{VE}$ values achieved in each set, in both groups. The difference between groups was only significant for $\mathrm{VO}_{2}$ on set $2(P<0.05)$, where the value was significantly higher for control subjects.

Borg symptom scores and physiological variables after the training session are shown in Table $3 . \mathrm{SpO}_{2}$ was significantly higher in control subjects after training than in participants with COPD $(P<0.05)$, but there were no other differences between groups for symptom scores. RT was well tolerated in all participants, and no adverse events occurred.

\section{Discussion}

The aim of this study was to assess the breath-by-breath response to one isokinetic RT session by measuring parameters of ventilation and gas exchange. The response was compared between people with COPD and healthy controls of a similar age. We did not know whether or not the ventilatory responses to RT would differ between groups, although one may anticipate that ventilatory limitations in patients with COPD would have an influence. Our data show that, although people with COPD worked at higher percentages of their maximum capacity, there were no statistically significant differences between the two groups for $\mathrm{VO}_{2}$ and $\mathrm{VE}$ achieved during training. However, controls were working at higher absolute $\mathrm{VO}_{2}$ and $\mathrm{VE}$ levels and were producing more quadriceps isokinetic work than people with COPD for each of the five RT sets ( $P<0.05$ between groups). The fact that people with COPD achieved lower quadriceps workloads at a higher proportion of their maximum ventilatory capacity suggests skeletal muscle dysfunction in this group. Although Table 1 indicates no difference in baseline isometric strength between the COPD and control groups, we found that, in the main RCT (larger numbers), isometric peak torque, isokinetic peak torque, and isokinetic total work were lower at baseline in people with COPD than in healthy controls. ${ }^{13}$

In healthy people, the response to acute strengthening exercises results in an increase in HR and BP, with minimal increases in $\mathrm{VO}_{2}$. As such, the cardiopulmonary adaptations to long-term resistance exercise tend to be minimal and vary depending on the specific strengthening exercise protocols. ${ }^{16,17}$ The intensity of effort parameters for improving cardiorespiratory fitness are well defined. The load of aerobic training should reach $50 \%-85 \%$ of maximum $\mathrm{VO}_{2}$ and/or $65 \%-85 \%$ of the maximal HR. ${ }^{18}$ In this study, we have witnessed a cardiorespiratory response to RT.

Two previous studies have examined the cardiorespiratory response to RT. The study by Sillen et $\mathrm{al}^{8}$ compared quadriceps RT (three sets of eight repetitions [ $70 \%$ of the one repetition maximum $\{1 \mathrm{RM}\}]$ ) with NMES in a cross-over study involving 13 subjects. This study also evaluated their results compared with a maximal cardiopulmonary exercise test (CPET) and therefore allows for easy comparison with the current study. Peak $\mathrm{VO}_{2}$ (\% of maximum from CPET) was $57 \%$ after RT and $34 \%$ after NMES $(P<0.001$ between $\mathrm{RT}$ and NMES). These findings are strikingly similar to those of the current study, where the percentage of maximum $\mathrm{VO}_{2}$ ranged from $49.1 \%$ to $60.1 \%$ after RT in participants with COPD. Peak VE (\% of maximum voluntary ventilation) was also comparable after RT in both the study by Sillen et $\mathrm{al}^{8}$ 
A

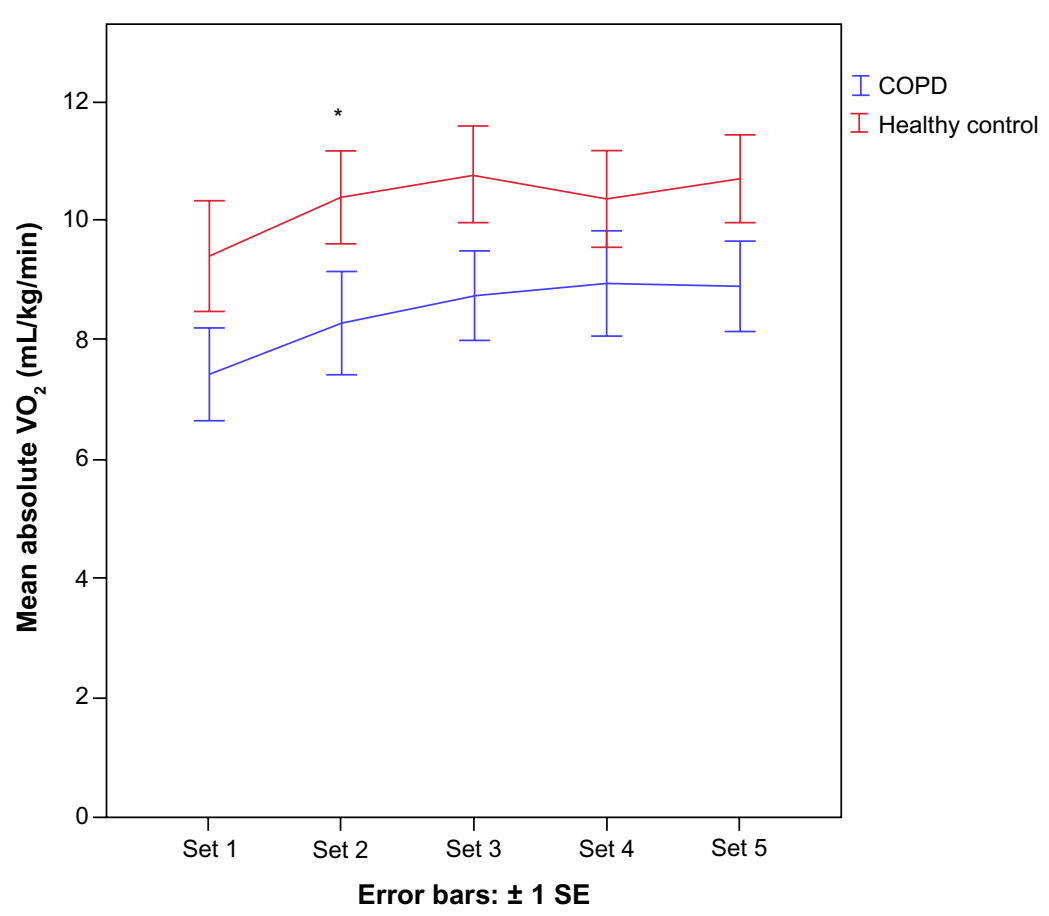

B

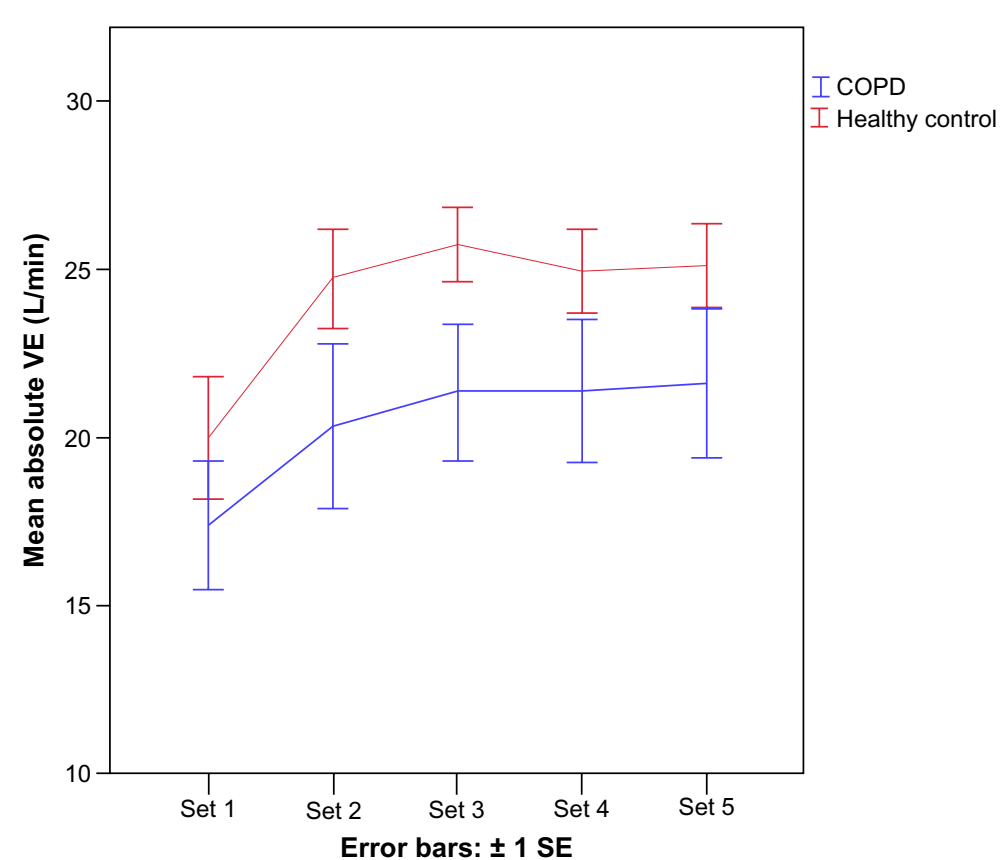

Figure I Mean actual oxygen consumption $(\mathbf{A})$ and ventilation $(\mathbf{B})$ achieved during sets $\mathrm{I}-5$ of a single isokinetic resistance training session in patients with chronic obstructive pulmonary disease and healthy controls.

Note: $* P<0.05$ significant difference between groups.

Abbreviations: COPD, chronic obstructive pulmonary disease; min, minutes; $\mathrm{SE}$, standard error; VE, ventilation; $\mathrm{VO}_{2}$, oxygen consumption.

$(58 \%)$ and in the current study, where the range was $57.6 \%-72.2 \%$ for people with COPD.

The second study, by Probst et al, ${ }^{7}$ is difficult to compare with the current study as the ventilation data are reported at three time points over a 12-week training period (week 1, 6, and 12) rather than a single session. Furthermore, the results are presented as actual values rather than related to a percentage of maximum from a previously reported peak test. Actual values for $\mathrm{VE}(\mathrm{L} / \mathrm{min}$ ) after leg press exercise ranged from 22 (week 1) to $26 \mathrm{~L} / \mathrm{min}$ (week 12). These findings are similar to those of the 
Table 3 Symptom and physiological variables after isokinetic resistance training

\begin{tabular}{lll}
\hline Characteristic & $\begin{array}{l}\text { COPD } \\
(\mathbf{n}=14)\end{array}$ & $\begin{array}{l}\text { Control } \\
(\mathbf{n}=1 \mathrm{I})\end{array}$ \\
\hline $\begin{array}{l}\text { Borg breathlessness score (0-I0) } \\
\text { [median (IQR)] }\end{array}$ & $3(\mathrm{I}-4)$ & $3(0-3)$ \\
Borg RPE score (6-20) [median (IQR)] & $13(12-15)$ & $13(12-15)$ \\
$\mathrm{HR}[$ mean (SD)] & $100.4(15.8)$ & $102.4(16.8)$ \\
$\mathrm{SpO}_{2}[$ mean (SD)] & $95.5(1.9)$ & $96.9(0.9)^{*}$ \\
\hline
\end{tabular}

Note: $* P<0.05$ between COPD and control subjects.

Abbreviations: COPD, chronic obstructive pulmonary disease; HR, heart rate; $\mathrm{IQR}$, interquartile range; RPE, rate of perceived exertion; SD, standard deviation; $\mathrm{SpO}_{2}$, oxygen saturation.

current study, as people with COPD had actual VE values of approximately $21 \mathrm{~L} / \mathrm{min}$ after sets 3-5 of RT. However, neither this study ${ }^{7}$ nor that of Sillen et $\mathrm{al}^{8}$ has compared the results in people with COPD with those of healthy, age-matched controls. Furthermore, this is the first study to report the cardiorespiratory cost of an isokinetic RT program.

There were some limitations with the analysis presented; primarily, that the sample size may have been too small to detect significant differences between the groups. Further study in a larger cohort is warranted. Healthy controls were significantly younger than patients with COPD; this may have had an impact on the results. Also, the ventilatory requirements of this training protocol were not directly compared with different modes of exercise. For instance, it would be interesting to see where this novel RT regime sits on the spectrum between typical endurance (eg, walking) and RT (eg, three sets of eight repetitions at $70 \%$ of $1 \mathrm{RM}$ ). Indeed, it would also be interesting to compare this training with some of the novel approaches proposed to reduce ventilatory demand (eg, single-leg cycling). ${ }^{19}$

Ventilatory and gas exchange measurements were taken at a standardized training session number (8-12 of 24 total sessions); it may have been interesting to take serial measures over the 8-week training period and to re-calibrate from a new CET, besides baseline. This would enable observation of whether the ventilatory load of the training changed in both groups during the 8-week training period. Finally, one may argue that the training protocol was not typical RT and was more akin to endurance training. It is likely that this type of training program sits somewhere on the spectrum between muscular endurance and $\mathrm{RT} .{ }^{20}$ However, the rapid training velocity was chosen as it was known to target type II muscle fibers, ${ }^{10}$ and subjects were asked to produce a maximal effort with each contraction.

The data in the current study show that the training was well tolerated in both groups. After RT, Borg breathlessness scores were a median of 3 ('moderate'), and RPE scores were 13 ('somewhat hard') in both groups. There were no significant differences between participants with COPD and controls for Borg scores after RT, therefore people with COPD did not perceive the training to be any harder than did healthy controls. However, control subjects were performing the RT at a higher absolute intensity. Borg scores after RT in the study by Sillen et $\mathrm{al}^{8}$ were similar to those in the current study: 3 for breathlessness and 3 for RPE (using the modified 0-10 Borg scale). No de-saturation in $\mathrm{SpO}_{2}$ was evident during RT for subjects with COPD. However, those on long-term oxygen had already been excluded from the wider study. If we consider that subjects with COPD had a mean HR after training of around 100 beats per minute (bpm), this relates to $67 \%$ of their maximum HR (220 minus mean age of the group [71 years]). Using the same formula, control subjects were working at $66 \%$ of their maximum HR. A HR of $65 \%-85 \%$ of maximum relates to the target training zone for cardiorespiratory fitness. ${ }^{21}$ It may be interesting to speculate that this type of training could therefore be expected to bring about central adaptations. Subjects in the study by Probst et $\mathrm{al}^{7}$ also had an HR approaching $100 \mathrm{bpm}$ after leg press exercise $(99,99$, and 104 at weeks 1, 6, and 12, respectively). However, HR may not be a useful indicator of training response in people with COPD and can be influenced by medications such as beta-blockers. ${ }^{22}$

The clinical implications of this work are that, whilst people with COPD worked at high percentages of their maximum ventilation, the Borg symptom scores remained at tolerable levels. This type of training may therefore be appropriate during a disease exacerbation, when patients are primary limited by breathless, ${ }^{23}$ as patients could have a cardiovascular training response without unacceptable symptoms. However, those with a recent disease exacerbation and those with comorbid conditions were excluded from the wider study. Therefore, we cannot directly infer that this type of training is safe and tolerable in these groups of patients, or whether the cardiopulmonary responses would be the same. However, Troosters et $\mathrm{al}^{24}$ have recently shown that RT is safe and successfully counteracts skeletal muscle dysfunction during acute exacerbations of COPD.

In addition, isokinetic training requires access to specialist equipment, which may be costly and not routinely available in clinical practice.

\section{Conclusion}

No statistically significant differences were found between people with COPD and healthy controls for $\mathrm{VO}_{2}$ and $\mathrm{VE}$ 
achieved during training. People with COPD performed RT at a significantly lower workload. The chosen RT program was performed comfortably by people with COPD, as Borg symptom scores remained at an acceptably low level after training and no oxygen de-saturation occurred. Whilst it may be that, in the most severe patients, we cannot overcome ventilatory limits, this RT protocol offers an attractive alternative training option that is well tolerated in people with stable COPD.

\section{Acknowledgments}

This work was supported by the Medical Research Council (MRC; grant ID 77170). The MRC had no role in the design, conduct, or analysis of the study.

The research took place at the National Institute for Health Research (NIHR) Collaboration for Leadership in Applied Health Research and Care (CLAHRC) based at the University Hospitals of Leicester National Health Service (NHS) Trust. The views expressed are those of the authors and not necessarily those of the NHS, the NIHR, or the Department of Health.

\section{Disclosure}

The authors report no real or perceived conflicts of interest in this work.

\section{References}

1. O'Shea SD, Taylor NF, Paratz J. Peripheral muscle strength training in COPD: a systematic review. Chest. 2004;126(3):903-914.

2. Spruit MA, Gosselink R, Troosters T, De Paepe K, Decramer M. Resistance versus endurance training in patients with COPD and peripheral muscle weakness. Eur Respir J. 2002;19(6):1072-1078.

3. O'Shea SD, Taylor NF, Paratz JD. Progressive resistance exercise improves muscle strength and may improve elements of performance of daily activities for people with COPD: a systematic review. Chest. 2009;136(5):1269-1283.

4. Bernard S, Whittom F, Leblanc P, et al. Aerobic and strength training in patients with chronic obstructive pulmonary disease. Am J Respir Crit Care Med. 1999;159(3):896-901.

5. O'Donnell DE. Ventilatory limitations in chronic obstructive pulmonary disease. Med Sci Sports Exerc. 2001;33(Suppl 7):S647-S655.

6. Simpson K, Killian K, McCartney N, Stubbing DG, Jones NL. Randomised controlled trial of weightlifting exercise in patients with chronic airflow limitation. Thorax. 1992;47(2):70-75.

7. Probst VS, Troosters T, Pitta F, Decramer M, Gosselink R1. Cardiopulmonary stress during exercise training in patients with COPD. Eur Respir J. 2006;27(6):1110-1118.
8. Sillen MJ, Janssen PP, Akkermans MA, Wouters EF, Wpruit MAl. The metabolic response during resistance training and neuromuscular electrical stimulation (NMES) in patients with COPD, a pilot study. Respir Med. 2008;102(5):786-789.

9. Gosselink R, Troosters T, Decramer M. Interactive course on exercise-based assessments in respiratory medicine. [Course notes]. European Respiratory Society. Lausanne. 2004:1-21.

10. Jones SW, Hill RJ, Krasney PA, O’Conner B, Peirce N, Greenhaff PL. Disuse atrophy and exercise rehabilitation in humans profoundly affects the expression of genes associated with the regulation of skeletal muscle mass. FASEB J. 2004;18(9):1025-1027.

11. Williams JEA, Flora KK, Sandland CJ, et al. Resistance training using an isokinetic dynamometer during pulmonary rehabilitation: a feasibility study. Am J Respir Crit Care Med. 2007;175:A854.

12. Robles PG, Mathur S, Janaudis-Fereira T, Dolmage TE, Goldstein RS, Brooks D. Measurement of peripheral muscle strength in individuals with chronic obstructive pulmonary disease: a systematic review. J Cardiopulm Rehabil Prev. 2011;31(1):11-24.

13. Constantin D, Menon MK, Houchen-Wolloff L, et al. Skeletal muscle molecular responses to resistance training and dietary supplementation in COPD. Thorax. 2013;68(7):625-633.

14. Quanjer PH, Tammeling GJ, Cotes JE, et al. Symbols, abbreviations and units. Working Party Standardization of Lung Function Tests, European Community for Steel and Coal. Eur Respir J Suppl. 1993;16:85-100.

15. Borg GA. Psychophysical bases of perceived exertion. Med Sci Sports Exerc. 1982;14(5):377-381.

16. Kispert CP, Nielsen DH. Normal cardiopulmonary responses to acute- and chronic-strengthening and endurance exercises. Phys Ther. 1985;65(12):1828-1831.

17. Hagberg JM, Graves JE, Limacher M, et al. Cardiovascular responses of 70- to 79-yr-old men and women to exercise training. J Appl Physiol (1985). 1989;66(6):2589-2594.

18. No authors listed. American College of Sports Medicine Position Stand. The recommended quantity and quality of exercise for developing and maintaining cardiorespiratory and muscular fitness, and flexibility in healthy adults. Med Sci Sports Exerc. 1998;30(6):975-991.

19. Dolmage TE, Maestro L, Avendano MA, Goldstein RS. The ventilatory response to arm elevation of patients with chronic obstructive pulmonary disease. Chest. 1993;104(4):1097-1100.

20. Kraemer WJ, Mazzetti SA, Ratamess NA, Fleck SJ. Specificity of training modes. In: Brown LE, editor. Isokinetics in Human Performance. Champaign (IL): Human Kinetics; 2000:25-41.

21. Tanaka H, Monahan KD, Seals DR. Age-predicted maximal heart rate revisited. J Am Coll Cardiol. 2001;37(1):153-156.

22. Aliverti A, Macklem PT. How and why exercise is impaired in COPD Respiration. 2001;68(3):229-239.

23. Rodriguez-Roisin R. Toward a consensus definition for COPD exacerbations. Chest. 2000;117(5 Suppl 2):398S-401S

24. Troosters T, Probst VS, Crul T, et al. Resistance training prevents deterioration in quadriceps muscle function during acute exacerbations of chronic obstructive pulmonary disease. Am J Respir Crit Care Med. 2010;181(10):1072-1077.
International Journal of COPD

\section{Publish your work in this journal}

The International Journal of COPD is an international, peer-reviewed journal of therapeutics and pharmacology focusing on concise rapid reporting of clinical studies and reviews in COPD. Special focus is given to the pathophysiological processes underlying the disease, intervention programs, patient focused education, and self management protocols.

\section{Dovepress}

This journal is indexed on PubMed Central, MedLine and CAS. The manuscript management system is completely online and includes a very quick and fair peer-review system, which is all easy to use. Visit http://www.dovepress.com/testimonials.php to read real quotes from published authors. 